\title{
COMPUTATIONAL STUDY OF CURVED UNDERBODY DIFFUSERS
}

\author{
Angel Huminic ${ }^{1, *}$, and Gabriela Huminic ${ }^{1}$ \\ ${ }^{1}$ Transilvania University of Brasov, Brasov 500036, Romania
}

\begin{abstract}
This paper presents new results concerning the aerodynamics of the Ahmed body fitted with a non-flat underbody diffuser. As in previous investigations performed, the angle and the length of the diffuser are the parameters systematically varied within ranges relevant for a hatchback passenger car. Coefficients of lift and drag are compared with the values obtained for the flat underbody diffuser, and the results reveal significant improvements concerning aerodynamic characteristics of body.
\end{abstract}

\section{Nomenclature}

$A \quad$ reference area of body

$c_{D} \quad$ drag coefficient

$c_{L} \quad$ lift coefficient

$c_{p} \quad$ pressure coefficient

$l_{d} \quad$ diffuser lehgt

$R^{2} \quad$ R-squared value of probability

$R e \quad$ Reynolds number

$p \quad$ pressure

$\mathrm{V} \quad$ air velocity

$x, y, z$ coordinates of the reference frame

$y^{+}$dimensionless wall coordinate

$\alpha_{d} \quad$ diffuser angle

$\delta$ deviation of CFD results from experiment

\section{Introduction}

Because aerodynamics contributes significantly to the performances of the road vehicles, designers pay recently much attention to the aero control packages of cars. For high-speed vehicles, these could consist on various aero elements, as wings, air splitters, flaps, side skirts, underbody diffusers etc. The later ones are recently widely used also for passenger cars due to the advantages they could generate: reducing of both drag and lift, which have a significant impact related to fuel consumption, aeroacoustics, stability and handling of vehicles.

Concerning aero elements, most of the studies performed have focused on the aerodynamics of cars' bodies fitted with simple plane underbody diffusers. As revealed by a recent review [1], significant knowledge has been already acquired in this field regarding the benefits induced by this device, also concerning the physics and phenomena which describe the flow through diffusers. Summarizing the conclusions of the previous studies, the air flow underneath cars with small ground clearances may generates the Venturi effect, namely a pressure fall in the constricted section between body and road, similarly to the flows through constricted tubes, as depicted in Figure 1, which reveals the pressure coefficient variation in the symmetry plane of a bluff body [2].

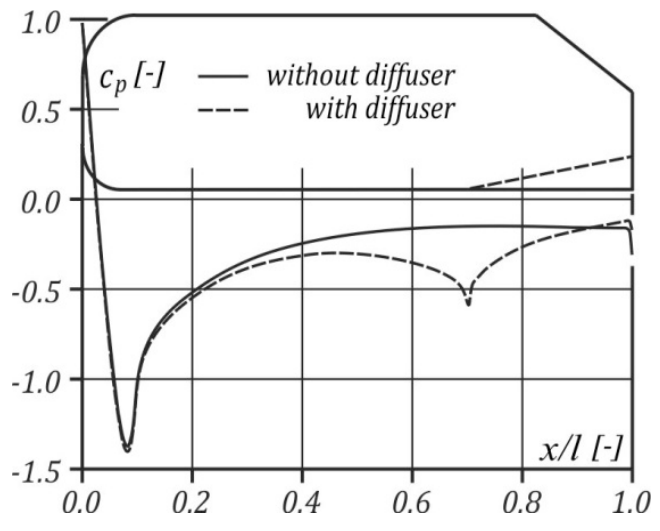

Figure 1

Fig. 1. Variation of pressure coefficient beneath a bluff body [2]

As shown, the flow accelerates in the first stage around the inlet of underbody, creating thus a depression, which is similar for both situations. It follows a pressure recovery beneath the body, back to the atmospheric pressure. This takes place continuously for a flat underbody, and in two stages for the underbody with diffuser, due to a new acceleration of the flow around the diffuser's first edge, which increases the depression and the downforce generated. Along the diffuser, a part of the kinetic energy of the fluid turns into potential pressure energy. This phenomenon is known as pumping effect [3]. In addition, the pressure drop at the exit is significantly smaller in the case of the underbody with diffuser. This contributes to smaller vortices and consequently to the decreasing of drag. Hence, diffusers may be used also to control boundary

\footnotetext{
* Corresponding author: angel.h@unitbv.ro
} 
layer separation of flow underneath cars, in order to avoid large turbulence phenomena behind them.

Following this basic idea, to increase the depression under cars' bodies, several recent studies have proposed new solutions concerning the geometry of these aero devices. Thus, Kekus and Angland [4] examined an Ahmed type body fitted with two-steps underbody plane diffuser, and Ehirim et al. [5] studied a plane underbody diffuser having a convex bump near the end. Using a generic body of car on wheels, Rossitto et al. [6] investigated the effect of a curved underbody diffuser for two configurations.

Being a continuous subject of research in the field of aerodynamics, current paper presents new results concerning the aerodynamics of the Ahmed body fitted with a non-flat underbody diffuser. As in previous investigation performed [7], the angle $\left(\alpha_{d}\right)$ and the length of the diffuser $\left(l_{d}\right)$ are the parameters systematically varied within ranges relevant for a hatchback passenger car. Coefficients of lift and drag are compared with the values obtained for the flat underbody diffuser, and the results reveal significant improvements concerning aerodynamics of body.

\section{Research Methodology}

For the current study, a computational approach was adopted, based on amount of work and number of models involved in investigation. Thus, several CFD analyses were performed using ANSYS CFX, which is a completely integrated CFD (Computational Fluid Dynamics) software. In the first stage, the accuracy and validity of the adopted computational model and procedure were checked using the experimental results of Strachan et al. [8]. The main stages of CFD simulations are described in the next paragraphs.

\subsection{CAD and computational models}

The simulations were carried out using the bluff body of Ahmed et al. [9], because its aerodynamics has been widely investigated in many studies and it is very well known. It is $1044 \mathrm{~mm}$ long, $389 \mathrm{~mm}$ wide and $288 \mathrm{~mm}$ height, and consists of three sections: a short curved one in front, followed by a long straight middle section and an angled $(\alpha)$ rear end. For current study $\alpha=35^{\circ}$ was adopted because it corresponds to a low drag and stable aerodynamic configuration. Thus, it represents a generic 1:4 scale model of lower-medium size hatchback vehicles, as depicted in Fig. 2, which show also the lateral view of the body used in current investigation. A circular curved underbody diffuser of radius $r_{d}$ was considered, with the beginning tangent $(T)$ to the flat surface of the underbody. Length $\left(l_{d}\right)$ of angle $\left(\alpha_{d}\right)$ of the diffuser are established as for plane diffuser.

The surfaces of the body were drawn with the aid of the CAD module of ANSYS, and integrated into a rectangular enclosure computational domain. Based on length $(l)$ of the body, it was such of dimensions that the adverse pressure effects between the body and the walls are negligible [2, 7]:
- $2.5 l$ in front of the body,

- $12 l$ behind the body,

- $1.5 l$ for top and lateral boundaries.

The body was placed $50 \mathrm{~mm}$ above the ground as in original study. Thus, the blockage ratio was smaller than $2 \%$, significantly smaller than in a wind tunnel test chamber.
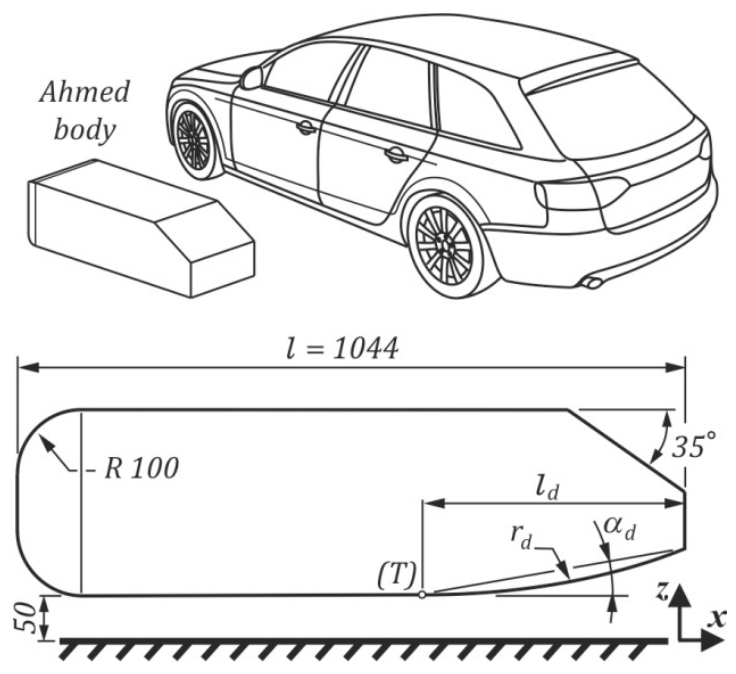

Fig. 2. Ahmed body, 3D view and lateral view (dimensions are in $\mathrm{mm}$ )

A multi-block scheme was used to generate the mesh of the computational domain. It consists on tetrahedral and hexahedral elements in proximity of the solid surfaces to solve accurately the flow inside the boundary layer, which consists of 30 layers. The first layer of cells around body was set to fulfill the condition $30 \leq y^{+} \leq$ 100 for computations using the "wall function" approach.

\subsection{Simulations setup and boundary conditions}

The used code solves the full Reynolds-Averaged Navier-Stokes equations in their conservation form: continuity equation and momentum equation. Transient analyses were performed for incompressible, adiabatic and fully turbulent flows (turbulence intensity of $0.2 \%$.) in conditions of the standard atmosphere: $p_{\infty}=$ $101325 \mathrm{~Pa}$ (reference pressure) and $t_{\infty}=15^{\circ} \mathrm{C}$ (temperature of the air). The solver uses these to compute density and viscosity of the air. Shear-StressTransport (SST) closure model of Menter [10] was employed to perform the simulations. The following boundary conditions were considered:

- uniform and constant velocity at inlet, $v_{x}=v_{\infty}$, $v_{y}=v_{z}=0$, according to the frame in Fig. 2, and also for ground, as for moving walls,

- a zero pressure condition, $p=0$, at the outlet boundary

- no slip conditions on the surfaces of the body, $v_{x}=v_{y}=v_{z}=0$, 
- free slip conditions for the top and lateral surfaces, as for fluid frontiers.

The reference velocity of air was set to $\mathrm{v}_{\infty}=40 \mathrm{~m} / \mathrm{s}$ as in the original study of Ahmed, which corresponds to a Reynolds number, $\operatorname{Re}=2.36 \cdot 10^{6}$, based on the length of the body.

Influence of the diffuser's parameters on drag and lift of body was investigated for the following angles $\alpha_{d}=$ $2^{\circ}, 4^{\circ}, 6^{\circ}, 8^{\circ}$ over a range of diffuser length $l_{d}=$ $(0.1,0.2,0.3,0.4) 1$, taking into consideration the diffuser height in road is limited due to the presence of the rear bumpers.

\subsection{Validation of CFD procedure}

Accuracy of the results provided by the computational model and CFD procedure used in the current study have been checked against the experimental results provided Strachan et al. [8] with moving ground (MG). Thus, several simulations were performed in same conditions employed in these experiments, and the results are depicted in Figures 3 and 4 , for all angles $(\alpha)$ of the upper slanted rear surface of the Ahmed body. Current CFD results concerning drag coefficient were also compared with the values of Graysmith [8] and Ahmed et al. [9] obtained for stationary ground (SG).

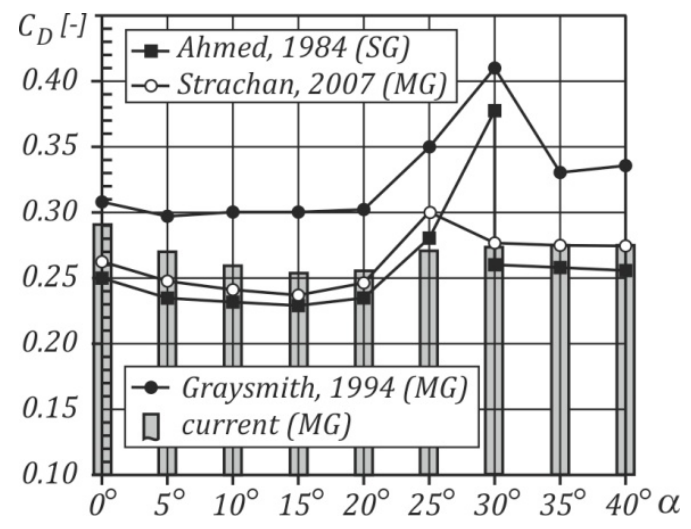

Fig. 3. Current results of $c_{D}$ against experiments $[8,9]$

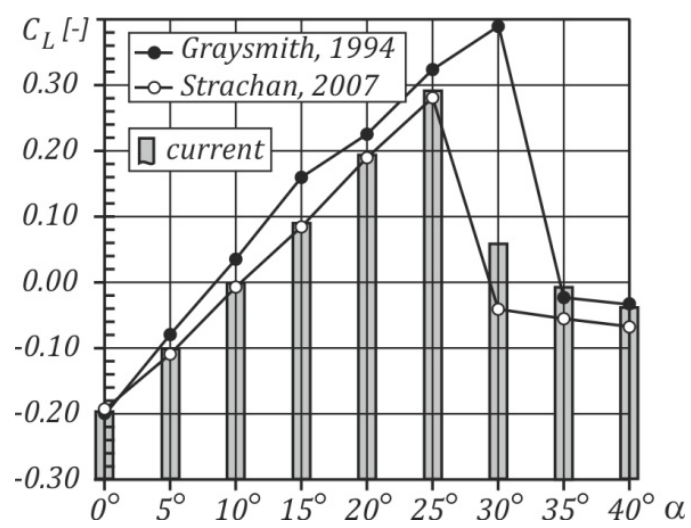

Fig. 4. Current results of $c_{L}$ against experiments $[8,9]$
As shown, the lift coefficients estimate closely the experiments of Stacthan for values of angle up to $\alpha=$ $25^{\circ}$ and the experiments of Graysmith for larger angles. Concerning the drag coefficient, there is a well prediction of Strachan experiment for $\alpha \geq 30^{\circ}$, when the flow over the angled surface becomes fully separated, including the case examined in current investigation of $\alpha=35^{\circ}$, for which deviation $(\delta)$ from experiment is smaller than $1 \%$,

$$
\delta=\frac{c_{\exp }-c_{C F D}}{c_{\exp }} 100[\%] .
$$

There were also examined the velocity contours in the wake using results provided by LDA (Laser Doppler Anemometry) investigation provided in [8], as revealed in Figure 5 for $v_{z} / v_{\infty}$ on $(y O z)$ plane, $(x / l)=0$ and $\alpha=25^{\circ}$.
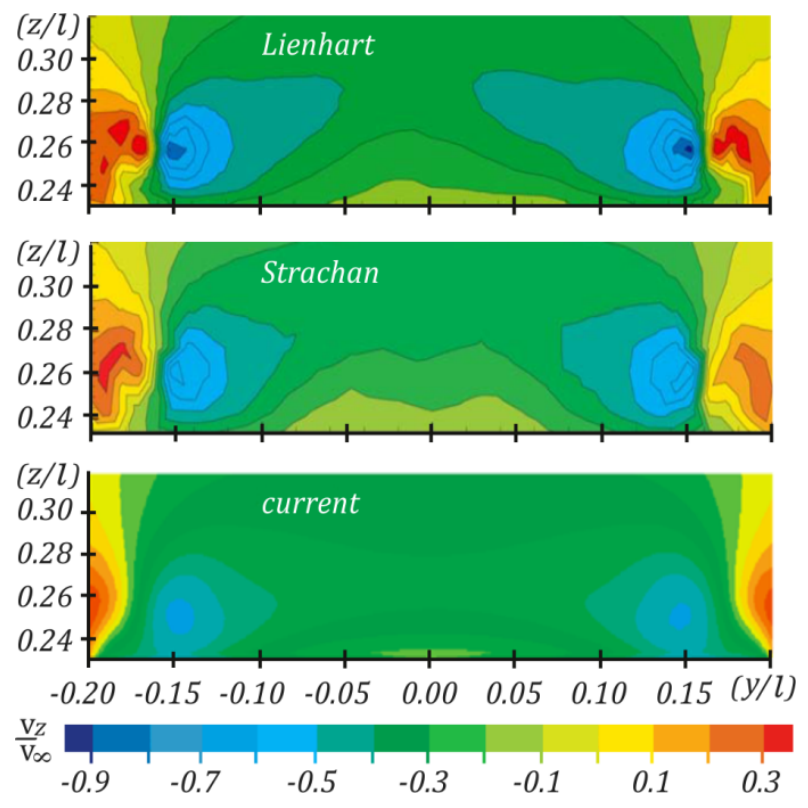

Fig. 5. Velocity contours $\mathrm{v}_{\mathrm{z}} / \mathrm{v}_{\infty}$ on $(y \mathrm{Oz})$ plane, $(x / l)=0$ and $\alpha=25^{\circ}[7]$

As depicted, the results are similarly for these investigations, all of them emphasizing the counterrotating lateral vortices which shed from the sides of the rear angled surface $[9,11]$.

\section{Results and Discussions}

In the first stage, the influence of plane diffuser was studied in order to have a reference. Then, the influence of the curved underbody diffuser was examined. Lift coefficient $c_{L}$ and drag coefficient $c_{D}$ were computed

$$
c_{L}=\frac{L}{\frac{\rho_{\infty} \mathrm{v}_{\infty}^{2}}{2} A}, c_{D}=\frac{D}{\frac{\rho_{\infty} \mathrm{v}_{\infty}^{2}}{2} A} .
$$


where $A=112032 \mathrm{~mm}^{2}$ is the reference area of the bluff body. Based on aerodynamic coefficients values, interpolation functions $c_{L}\left(\alpha_{d}, l_{d}\right)$ and $c_{D}\left(\alpha_{d}, l_{d}\right)$ were found $\left(R_{\min }^{2}=0.9988\right)$ as depicted in Figures 6 and 7. Surfaces with grid correspond to the case of curved underbody diffuser.

As shown, there is a substantial growth of the downforce generated, the lift coefficient of body with curved diffuser being significantly lower than that of body with plane diffuser. Lift decreases continuously with both diffuser angle and length reaching the following minimum values: $c_{L}=-0.645$ for the plane diffuser and $c_{L}=-0.814\left(\Delta c_{L}=26.2 \%\right)$. The average decreasing of lift for all configurations studied is $\Delta c_{L}=$ $22 \%$.

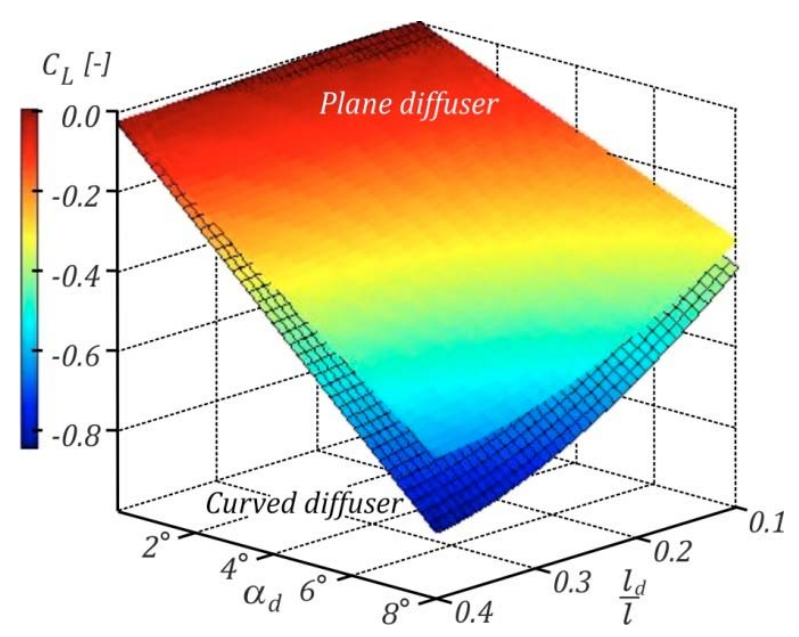

Fig. 6. 3D maps of $c_{L}\left(\alpha_{d}, l_{d}\right)$ for plane diffuser (surface without grid) and curved diffuser (surface with grid)

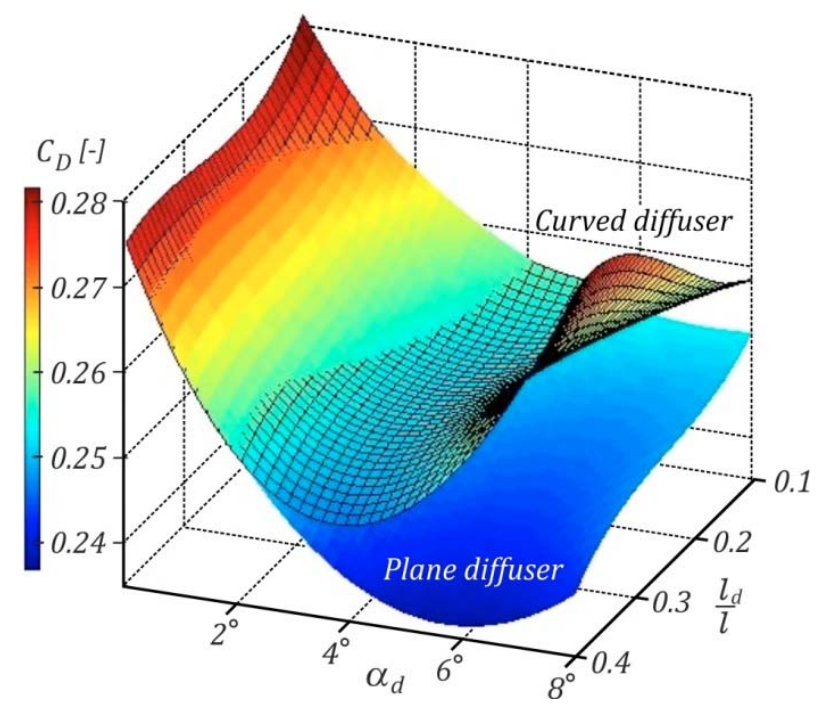

Fig. 7. 3D maps of $c_{D}\left(\alpha_{d}, l_{d}\right)$ for plane diffuser (surface without grid) and curved diffuser (surface with grid)

Concerning drag, the results show a more complex variation with diffuser parameters. Thus, for small angles $\alpha_{d}$, the drag decreases and reaches a minimum value, faster for curved diffuser, then it starts to rise. As depicted in Figure 7, for angles $\alpha_{d}=\left(2^{\circ}, 4^{\circ}\right)$ the curved diffuser could also generates smaller values of drag, as similarly as for lift force.

Hence, shorter diffusers with curved section works better from aerodynamic point of view, as revealed in Figure 8. For current investigation, the minimum values of drag found are $c_{D}=0.237$ for the flat diffuser $\left(\alpha_{d}=\right.$ $6^{\circ}$ and $\left.l_{d}=0.4 l\right)$ and $c_{D}=0.246\left(\alpha_{d}=3^{\circ}\right.$ and $l_{d}=$ $0.4 l)$ in the case of of the curved diffuser.

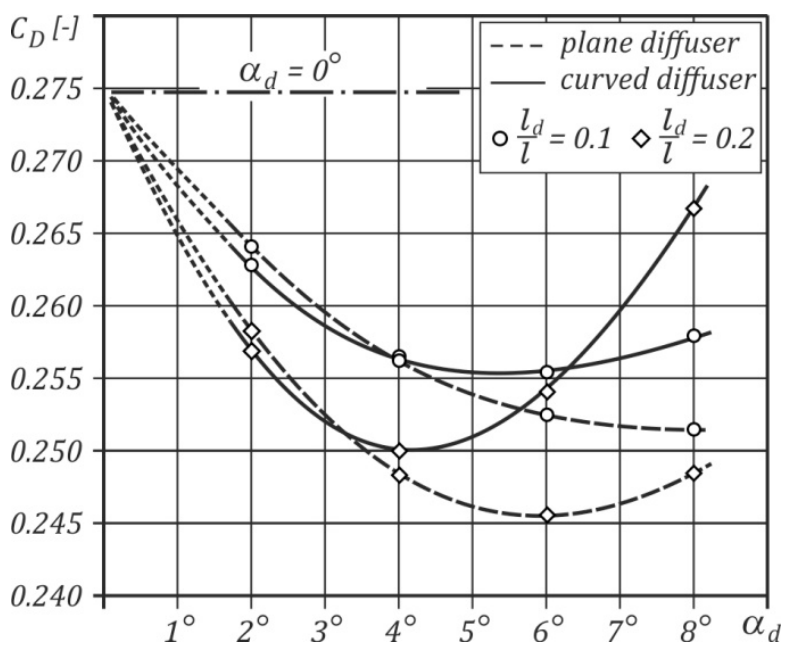

Fig. 8. Variation of $c_{D}\left(\alpha_{d}\right)$ for $l_{d}=(0.1,0.2) l$

Figure 9 shows also a two stage of pressure recovery beneath the body. Since there is no edge (singularity) that generates a sudden increase in speed, pressure variation occurs continuously. The cusp point of $c_{p}$ variation turns into an inflexion point and the depression increases under body, which leads also to a larger downforce generated by the curved diffuser.

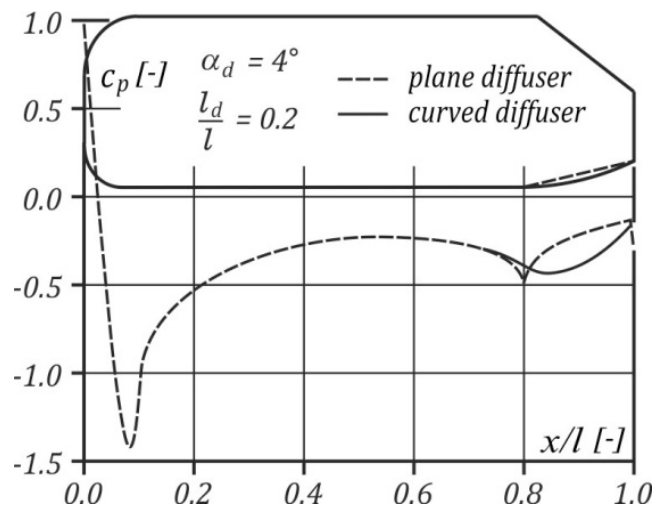

Fig. 9. Variation of $c_{p}$ in the symmetry plane for $\alpha_{d}=4^{\circ}$ and $l_{d}=0.2 \mathrm{l}$

\section{Conclusions}

In this paper, a bluff body with circular curved underbody diffuser is studied, through the variation of its parameters, length and angle. The values obtained are compared with those of the plane diffuser. The results reveal an average growth $\Delta c_{L}=22 \%$ of the downforce generated using the curve diffuser. In addition, for small 
diffuser length, $l_{d}<0.2 l$ and moderate angle $\alpha_{d}=$ $\left(2^{\circ}, 4^{\circ}\right)$, the curved diffuser leads also to smaller values of drag. Thus, shorter diffusers with curved section works better from aerodynamic point of view.

\section{References}

1. A. Mecke, I. Lee, J.R. Baker jr., M.M. Banaszak Holl, B.G. Orr, Eur. Phys. J. E 14, 7 (2004)

2. M. Ben Rabha, M.F. Boujmil, M. Saadoun, B. Bessaïs, Eur. Phys. J. Appl. Phys. (to be published)

3. Luigi T. De Luca, Propulsion physics (EDP Sciences, Les Ulis, 2009)

4. F. De Lillo, F. Cecconi, G. Lacorata, A. Vulpiani, EPL, 84 (2008)

5. Ehirim O.H., Knowles K., Saddington A., 2018, A Review of Ground-Effect Diffuser Aerodynamics, J. of Fluids Engineering, 141, 020801 (19 pages)

6. Huminic A., Huminic G., Soica, A., 2012, Study of aerodynamics for a simplified car model with the underbody shaped as a Venturi nozzle, Int. J. of Vehicle Design, 58 pp. 15-32

7. 3Cooper K., Bertenyi T., Dutil G., Syms, J., 1998, The Aerodynamic Performance of Automotive Underbody Diffusers, Developments in Vehicle Aerodynamics SAE SP-1318, 5-36

8. 4. Kekus P., Angland D., 2018, Automatic Wind Tunnel-Based Optimisation of an Automotive Underbody Diffuser, AIAA Aerospace Sciences Meeting, doi: 10.2514/6.2018-0045

9. 5. Ehirim O.H., Knowles K., Saddington A.J., Finnis M.V., 2018, Aerodynamics of a Convex Bump on a Ground-Effect Diffuser, J. of Fluids Engineering, 140, 091102 (11 pages)

10. 6. Rossitto G., Sicot C., Ferrand V., Borée J., Harambat F., 2017, Aerodynamic performances of rounded fastback vehicle, Pro. of the Institution of Mechanical Engineers, Part D: J. of Automobile Engineering, 231, pp. 1211-1221

11. 7. Huminic A., Huminic G., 2017, Aerodynamic study of a generic car model with wheels and underbody diffuser, Int. J. of Automotive Technology, 18, pp. 397-404

12. 8. Strachan, R., Knowles, K., Lawson, N., 2007, The vortex structure behind an Ahmed reference model in the presence of a moving ground plane, J. of Experiments on Fluids, 42, pp. 659-669.

13. 9. Ahmed S., Ramm G., Faltin G., 1984, Some salient features of the time-averaged ground vehicle wake", SAE Technical Paper 840300

14. 10. Menter F.R., 1994, Two-equation eddy-viscosity turbulence models for engineering applications, AIAA Journal, 32, pp.1598-1605

15. 11. Guilmineau, E., 2008, Computational study of flow around a simplified car body, J. of Wind and Industrial Aerodynamics, 96, pp 1207-1217 